\title{
What's in the box? Authentication of Echinacea herbal products using DNA metabarcoding and HPTLC
}

4 Ancuta Cristina Raclariu ${ }^{\mathrm{a}, \mathrm{b}}$, Carmen Elena Ţebrencu ${ }^{\mathrm{c}, \mathrm{d}}$, Mihael Cristin Ichim ${ }^{\mathrm{b}}$, Oana 5 Teodora Ciupercăc ${ }^{\mathrm{c}}$, Anne Krag Brysting ${ }^{\mathrm{e}}$, Hugo de Boer, ${ }^{\mathrm{a}, *}$

${ }^{a}$ Natural History Museum, University of Oslo, P.O. Box 1172 Blindern, 0318 Oslo, 8 Norway.

$9 \quad{ }^{\mathrm{b}}$ Stejarul Research Centre for Biological Sciences, National Institute of Research and 10 Development for Biological Sciences, Alexandru cel Bun Street, 6, 610004 Piatra 11 Neamt, Romania.

$12{ }^{\mathrm{c}}$ Research and Processing Center for Medicinal Plants Plantavorel S.A., Cuza Voda 13 Street, 46, 610019, Piatra Neamt, Romania.

14 Academy of Romanian Scientists, Splaiul Independentei, 54, 050094, Bucharest, 15 Romania.

16 e Department of Biosciences, Centre for Ecological and Evolutionary Synthesis 17 (CEES), University of Oslo, P.O. Box 1066 Blindern, 0316 Oslo, Norway.

$19 *$ Corresponding author:

20 Hugo de Boer, Natural History Museum, University of Oslo, P.O. Box 1172 Blindern,

210318 Oslo, Norway

22 Tel.: +47 22851875.

23 E-mail address: hugo.deboer@nhm.uio.no (H. de Boer) 
Abstract

27 Background

Differences in regulatory policies between countries as well as a lack of appropriate standardized methods for authentication and quality control of herbal products lead to concerns over quality and safety. Echinacea products are among the top selling herbal products in Europe and the United States with indications for broad range of ailments.

33 Purpose

34 This study approached the need for a novel analytical strategy in authentication of 35 herbal products.

Methods

A combination of high performance thin layer chromatography (HPTLC) and DNA metabarcoding was employed. Fifty-three Echinacea herbal products marketed across Europe were tested to evaluate the accuracy of these methods in plant identification and their potential in detecting substitutes, adulterants and other unreported plant constituents.

Results

HPTLC provides a high resolution in detecting Echinacea phytochemical target compounds, but does not offer information on the other species within the product. Alternatively, we showed that the limitation of HPTLC to detect non-targeted species can be overcome through the complementary use of DNA metabarcoding. Using DNA metabarcoding, Echinacea species were detected in 34 out of the 38 retained products $(89 \%)$, but with a lack of discriminatory resolution at the species level due to the low level of molecular divergence within the Echinacea genus. All herbal products showed considerable discrepancies between ingredients listed on the label and the ones detected using DNA metabarcoding registering an overall ingredient fidelity of $43 \%$.

Conclusion

57 The results confirm that DNA metabarcoding can be used to test for the presence of Echinacea and simultaneously to detect other species present in even highly processed and multi-ingredient herbal products.

Keywords: DNA metabarcoding, Echinacea, herbal pharmacovigilance, herbal products, HPTLC

\section{Abbreviations}

EMA, European Medicines Agency; BLAST, basic local alignment search tool; FTIR Fourier-transformed infrared spectroscopy; IR, infrared spectrometry; HPTLC, high performance thin layer chromatography; mat K, maturase K; MS, mass spectrometry; MOTU, molecular taxonomic unit; nrITS, nuclear ribosomal internal transcribed spacer; Ph.Eur., European Pharmacopoeia; $r b c \mathrm{~L}$, ribulose bisphosphate carboxylase; TLC, thin-layer chromatography; UV-VIS, ultraviolet-visible. 


\section{Introduction}

\subsection{Legislation and Echinacea}

The regulation of herbal products varies globally, and products can be classified as food supplements, medicines, homeopathic products, cosmetics or even biocides depending on specific legislation. The European Medicines Agency (EMA) regularly produces updates of the European Pharmacopoeias (EDQM, 2014) and in addition has produced a number of monographs on quality and authentication of specific herbals providing relevant methodological specifications for their quality assessment. However, the primary legal responsibility for the safety of the marketed products is delegated by default to the manufacturers.

Sales of herbal dietary supplements have seen year-on-year increases of 5-10 $\%$, and in 2014 sales reached an estimated total of more than $\$ 6.4$ billion, with Echinacea among the top five top grossing taxa (Smith et al., 2015). Echinacea is a genus of composites (Asteraceae) comprising a small number of herbaceous grasslands perennial taxa whose natural distribution is limited to North America (Kindscher and Wittenberg, 2016). Three Echinacea species, E. angustifolia DC. (Narrow-leafed purple coneflower), E. pallida (Nutt.) Nutt. (Pale purple coneflower) and E. purpurea (L.) Moench (Purple coneflower), are used in traditional herbal medicine (EDQM, 2014).

Today Echinacea is cultivated widely in Europe and North America for use in commercial herbal products in a diverse range of products, including herbal teas, capsules, tablets, powders, tinctures and beverages (Brown et al., 2011). A 2007 survey by the National Centre for Complementary and Alternative Medicine showed that Echinacea was the most commonly used herbal medicine among adults and children in United States (Barnes et al., 2008). In Europe, a study on the use of food supplements and medicines showed that Echinacea and Ginkgo are the most common herbals (EAS, 2006).

\subsection{Pharmacological use and effects of Echinacea species}

Echinacea has been extensively studied for its pharmacological effects in both in vitro and in vivo studies (Table S1). In vivo human clinical studies on the oral administration of E. purpurea commercial herbal juice have failed to confirm previous in vitro findings (Schwarz et al., 2005, 2002; Sperber et al., 2004). However, a recent study conducted on 68,522 Norwegian women and their children revealed no risk of malformations or adverse pregnancy outcomes associated with the use of Echinacea in pregnancy (Heitmann et al., 2016).

\subsection{Chemical profiles and differences between Echinacea species}

The constituents of Echinacea species used for medicinal purposes differ qualitatively and quantitatively (Barnes et al., 2005; Binns et al., 2002). Alkamides, polysaccharides, glycoproteins, volatile oils and phenolic compounds have been considered important constituents of the plant (Table S2). Echinacea purpurea shows a similar phenolic phytochemical profile (cichoric acid, caftaric acid, chlorogenic acid) in roots, flower and leaves, but with no cynarine in the aerial parts, whereas $E$. angustifolia roots shows low amounts of cichoric acid and cynarine, and that of $E$. pallida shows both cichoric acid and cynarine. Echinacea purpurea aerial parts contain no echinacoside, while echinacoside is a major component in the roots of $E$. pallida and E. angustifolia (and also present in small quantities in the flower and leaves Alkamides are found in the rhizomes and roots of E. angustifolia, and less 
abundantly in the aerial parts of E. purpurea and mainly absent in E. pallida roots). E. pallida contains large amounts of ketoalkenes. Rutoside is a flavonoid present in the leaves of all the three species of Echinacea (Barnes et al., 2005).

\subsection{Quality issues of Echinacea herbal products}

The increased use of Echinacea species has led to concerns about adulterated products resulting from challenges in morphology-based identification, due to overlapping morphological variability and frequent hybridization between species (Flagel et al., 2008). Furthermore, reported adulteration of E. purpurea with the roots of Parthenium integrifolium L., Lespedeza capitata Michx., Eryngium aquaticum L., Rudbeckia nitida Nutt., Helianthus annuus L. or Liatris aspera Michx. lead to safety concerns of the herbal products (Zhang et al., 2017). The use of unreported ingredients is a serious safety concern as adverse drug reactions cannot be associated to the product label and ingredients (Gilbert, 2011). Commercially available herbal products contain one or more Echinacea species originating from the same or different geographical areas, and the resulting phytochemical diversity across these products can complicate further investigations (Barnes et al., 2005). Echinacea secondary metabolites are used for the qualitative identification of species in the industry. Presence of for example, cichoric acid for E. purpurea and E. angustifolia, echinacoside for E. angustifolia and E. pallida, ketoalkene for E. pallida were routinely tested (Mistrikova and Vaverkova, 2006). Nevertheless, the presence of these markers does not provide unequivocal identification of the species since, for instance, traces of echinacoside can be also found in E. purpurea (Arnason et al., 2002). Presently, the differentiation of species is based on the relative abundances of metabolites by various phytochemical techniques, but marketed herbal products are often highly processed complex formulations with numerous ingredients, and these methods might not enable accurate identification of all plant ingredients, especially if target species are admixed with other species (De Boer et al., 2015).

\subsection{DNA metabarcoding of herbal products and Echinacea}

High phenotypic plasticity within Echinacea has complicated the taxonomy of the genus and led to misidentifications, confused taxonomies and misapplication of taxa (Kindscher and Wittenberg, 2016). Combined plastid (trnS and trnG) and nuclear (Adh, CesA, and GPAT) phylogenies found no resolved topologies, suggesting incomplete lineage sorting, as well as the potential for widespread hybridization and backcrossing following secondary contact within the genus (Flagel et al., 2008). Flagel et al. (2008) note that in contrast to the low discriminatory resolution of these molecular markers, previous studies using morphological characters and metabolic profiles found well-resolved and taxonomically supported relationships within Echinacea, and suggest the use of markers with more broad genomic coverage. Zhang et al. (2017) recovered the complete plastid genomes from all nine Echinacea species, and based on both coding and non-coding regions of the chloroplast genomes, Echinacea species were separated into two clades with strong support. In addition, Zhang et al. (2017) report that the core barcoding markers mat $\mathrm{K}$ and $r b c \mathrm{~L}$ do not differ sufficiently for species-specific identification of Echinacea but suggest a combination of nrITS and trnH-psbA as the optimal barcoding markers instead. The incongruence in nuclear ribosomal and plastid phylogenies, reported by Flagel et al. (2008), however suggests that neither plastid markers nor nrITS provide an accurate picture of the phylogenetic history of the genus. 
In this study, we use DNA metabarcoding to detect species diversity in Echinacea herbal products. The use of DNA metabarcoding for the identification of commercialized plant products has evolved with advances in molecular biology and sequencing (Coghlan et al., 2012; Raclariu et al., 2017b), and is defined as highthroughput multispecies (or higher-level taxon) identification using the total and typically degraded DNA extracted from an environmental sample (Taberlet et al., 2012). Here we test the hypothesis that Echinacea species are frequently admixed with other species in Echinacea herbal products. We test this hypothesis by authenticating European Echinacea herbal products using DNA metabarcoding and HPTLC to authenticate these using phytochemical constituents, and aim to answer the following research questions: 1) Can DNA metabarcoding be used to test for the presence of Echinacea species in herbal products, and to detect the presence of off label plant species due to substitution or adulteration?; 2) Can HPTLC be used to distinguish Echinacea species and to identify its exclusive presence in herbal products?

\section{Materials and Methods}

\subsection{Sample collection}

Fifty-three herbal products listing different Echinacea species, marketed as single (35) and multi-ingredient (18) food supplements consisting in herbal teas (9 loose teas and 8 bagged teas), capsules (16), tablets (13) and extracts (7), were purchased from different retail stores (20), pharmaceutical companies (32) and via e-commerce (1). The countries of origin of these products were Austria (1), China (1), Czech Republic (3), France (1), Germany (4), Italy (2), Macedonia (1), Poland (2), Romania (26), Switzerland (1), United States (7), and four of the products did not specify a country of origin. A list of samples is included as Table S3, but the producer/importer name, lot number, expiration date and any other information that could identify the specific products and producers are omitted. These herbal products were imported into Norway for scientific analysis under Norwegian Medicines Agency license no. $16 / 04551-2$.

\subsection{High performance thin layer chromatography (HPTLC) analysis}

Echinacea phenylpropanoids (echinacoside, cynarin, cichoric acid, chlorogenic acid, caffeic acid, caftaric acid) from 53 herbal products were analyzed using HPTLC following recommendations in the specific monographs from the European Pharmacopoeia $8^{\text {th }}$ Edition (EDQM, 2014) and the CAMAG application notes on HPTLC identification of Echinacea species (2015). Herbal products were processed depending on their pharmaceutical formulation. Capsules (about $300 \mathrm{mg}$ ), tablets (400 $\mathrm{mg}$ ), and teas $(500 \mathrm{mg})$ were homogenized and mixed in a 1:100 ratio with methanol/water $(80: 20 \mathrm{~V} / \mathrm{V})$ in an ultrasonic bath for $15 \mathrm{~min}$, and tinctures were diluted in 1:3 methanol. The obtained solutions were centrifuged and the filtered supernatant was collected and used as sample for further analysis. The following chemical and botanical standards were used. Reference substances: caftaric acid (HPLC grade, Carl Roth GmbH + Co. KG, Karlsruhe, Germany), cynarine (min. 98.0 $\%$, HPLC/Sigma-Aldrich Co, St. Louis, Missouri, USA), cichoric acid (min. 95.0\%, Sigma-Aldrich, St. Louis, Missouri, USA), echinacoside (min. 98.0 \% HPLC, SigmaAldrich Co, St. Louis, Missouri, USA), chlorogenic acid (min. $95.0 \%$, SigmaAldrich), caffeic acid (TLC grade, Carl Roth GmbH + Co. KG, Karlsruhe, Germany), 
verbascoside (min. $99.0 \%$ HPLC, Extrasynthese SAS, Lyon, France), ferulic acid (min. 99.0 \%, Sigma-Aldrich, St. Louis, Missouri, USA). Solvents: ethanol, min. 96 $\% \mathrm{~V} / \mathrm{V}$, and methanol, $99.3 \%$ analytical reagent (Chimreactiv SRL, Bucharest, Romania). Analytical reagents: formic acid ACS (Merck KGaA, Darmstadt, Germany), ethyl acetate (min. 99.5 \%, Sigma-Aldrich Co, St. Louis, Missouri, USA), distilled water prepared in laboratory; natural products - polyethylene glycol (NP/PEG) reagent (Honeywell Fluka, Seelze, Germany). Botanical standards: United States Pharmacopoeia (USP) analytical reference botanical standards: USP Powdered Echinacea purpurea F0D018, USP Powdered Echinacea angustifolia G01377 and USP Powdered Echinacea pallida F0I285 (Rockville, Maryland, USA). Apparatus: A CAMAG high performance thin layer chromatography system (CAMAG AG, Muttenz, Switzerland), with a Linomat IV sample applicator, a Canon digital camera, and the following software, Reprostar III with winCATS planar chromatography manager software, Digistor II digital system with winCATS software with an Image Comparison Viewer enabling high resolution visualization of multiple samples for comparison of specific compound retention times and the included references.

Samples and references were analyzed on Silica gel HPTLC plates (60 F245 20x10 and 10x10 cm for tinctures and solutions, Merck KGaA, Darmstadt, Germany). The reference solutions were dissolved in methanol to obtain the following concentrations: caftaric acid $0.006 \%$, cynarine $0.02 \%$, cichoric acid $0.005 \%$, echinacoside $0.018 \%$, chlorogenic acid $0.015 \%$, caffeic acid $0.011 \%$, verbascoside $0.01 \%$, ferulic acid $0.01 \%$, USP Powdered Echinacea purpurea F0D018 1.0 \%, USP Powdered Echinacea angustifolia G0I377 $2.0 \%$ and USP Powdered Echinacea pallida F0I285 $1.0 \%$. Echinacea product sample extracts and reference solutions were applied separately in twin bands, on maximum 12 tracks at 4-8 mm distance using different application volumes (4-16 $\mu 1$ for extracts, 12-17 $\mu$ l for bag and bulk teas, 12-17 $\mu \mathrm{l}$ for tablets, 9-15 $\mu \mathrm{l}$ for capsules and 3-6 $\mu \mathrm{l}$ for standard solutions) with a band length of $8-10 \mathrm{~mm}$ and a delivery speed of $8 \mathrm{~s} / \mu 1 \mathrm{using}$ a CAMAG Linomat IV automatic sample applicator (see above). The plates were run for $30 \mathrm{~min}$ in the developing box and subsequently dried at $105{ }^{\circ} \mathrm{C}$ for $5 \mathrm{~min}$. For phenylpropanoids identification, the plates were inspected at 254 and $366 \mathrm{~nm}$ before and after homogenous spraying with the Natural products-polyethylene glycol reagent (NP/PEG), and subsequently air dried. For each set of samples, the plates were developed in a saturated vertical-developing chamber at room temperature $\left(20-22{ }^{\circ} \mathrm{C}\right)$ for $30 \mathrm{~min}$ with ethyl acetate:formic acid:acetic acid:water $=20: 2.2: 2.2: 5.4 \mathrm{~V} / \mathrm{V}$ as mobile phase; the development distance was $7 \mathrm{~cm}$; after the development, the plates were air dried at room temperature. Derivatization of the chromatograms were performed by spraying the plates with NP $(0.1 \mathrm{~g}$ in $10 \mathrm{ml}$ methanol) and PEG 400 $\left(0.5 \mathrm{~g}\right.$ in $10 \mathrm{ml}$ ethanol), followed by heating of the plates at $105^{\circ} \mathrm{C}$ temperature for 15 min. Plates were subsequently imaged using a CAMAG Reprostar 3 with digital video camera at $254 \mathrm{~nm}$ for developed plates and $366 \mathrm{~nm}$ for derivatized plates. HPTLC densitometry (CAMAG- TLC Scanner 3 with WinCATS Planar Chromatography Manager Software) was done using spectra recording from 200-700 $\mathrm{nm}$, absorption at $254 \mathrm{~nm}$ wavelength, slit dimension $8.00 \times 0.40 \mathrm{~mm}$, scanning speed $100 \mathrm{~nm} / \mathrm{s}$, and peak area evaluation by linear or polynomial regression.

\subsection{DNA metabarcoding}

The total DNA was extracted from small amounts (about $300 \mathrm{mg}$ ) of each homogenized herbal product or silica gel dried leaves, using the method described in Raclariu et al. (2017b). Amplicon DNA metabarcoding using barcoding markers 
nrITS1 and nrITS2 was done using the exact method described in Raclariu et al. (2017b), and sequenced on an Ion Torrent Personal Genome Machine (Life Technologies, Thermo-Fischer Scientific, USA). Sequencing read data was processed using the HTS barcode-checker pipeline (Lammers et al., 2014) as described in Raclariu et al. (2017b). A $99 \%$ sequence similarity threshold was used for MOTU clustering with a minimum of 10 reads per cluster to reduce the formation of false MOTUs and the potential effects of sequencing bias known to affect the Ion Torrent sequencing platform (Loman et al., 2012). One representative sequence from each MOTU was taxonomically assigned using the Basic Local Alignment Search Tool (BLAST) (Altschul et al., 1990) against a reference nucleotide sequence database represented by a local copy of the NCBI/GenBank. BLAST results with a maximum e-value of 0.05 , a minimum hit length of $100 \mathrm{bp}$, and similarity of $>99 \%$ against the reference barcode were accepted as species level matches.

\section{Results}

\subsection{High performance thin layer chromatography (HPTLC)}

Identification and detection of Echinacea species, E. purpurea, E. angustifolia and E. pallida, from tea, tablets, capsules, and extracts were done using HPTLC with the standard Echinacea phenylpropanoid references echinacoside, cichoric acid, caftaric acid, chlorogenic acid, and cynarine, and the botanical standards of E. purpurea, E. angustifolia, E. pallida. In the HPTLC chromatograms visualized at $366 \mathrm{~nm}$ after derivatization, the occurrence of fluorescent blue spots indicates the presence of phenylpropanes (Figure 1). The phenylpropanoid refraction (Rf) values were: 0.15 0.17 (echinacoside), 0.47-0.49 (chlorogenic acid), 0.51-0.53 (caftaric acid), 0.65 (cynarine), 0.87-0.9 (cichoric acid), 0.96-0.97 (caffeic acid), and other polyphenols (ferulic acid and verbascoside) at $\mathrm{Rf}=0.35 ; 0.46 ; 0.52 ; 0.73$ and 0.90 .

[INSERT] Figure 1. High performance thin layer chromatogram (HPTLC) based identification and detection of phenylpropanoids from the Echinacea sp. herbal products. The track assignment: (1) test solutions (1-53) (2) phenylpropanoids chemical reference solutions in the following order (increasing Rf): echinacoside, cholorogenic acid, caftaric acid, cynarin, cichoric acid, caffeic acid; (3) botanical reference solution of Echinacea purpurea (root (MNR1) and aerial parts (MH1)) (4) United States Pharmacopoeia (USP) analytical reference botanical standards ( $E$. purpurea (Spu), E. angustifolia (SA) and E. pallida (SP)) A. HPTLC chromatograms of herbal teas. B. HPTLC chromatograms of capsules. C. HPTLC chromatograms of tablets. D. HPTLC chromatograms of extracts. The chromatograms were scanned after derivatization in UV $366 \mathrm{~nm}$. Details about the herbal products can be found in the Table S3.

The results of HPTLC chromatographic identifications of the analysed herbal teas yielded the following results for the 10 samples labelled as including Echinacea sp. (Figure 1A and Table S4): Sample \#1 doesn't contain any of the targeted phenylpropanoids specific to Echinacea species; sample \#8 has caffeic acid and low caftaric acid and content suggesting that only E. purpurea is present and in low concentration; sample \#12 has very strong zones for echinacoside, cichoric acid and caftaric acid indicating a mixture of E. purpurea and E. pallida; seven samples (samples \#4, \#6, \#9, \#10, \#11,\#13, and \#14) show distinct presence of caftaric and 
cichoric acid indicating presence of E. purpurea. The six samples labelled as including specifically $E$. purpurea yielded the following: in four samples (samples \#3, $\# 15, \# 16, \# 17$ ) the presence of this species was confirmed by high content of caftaric and cichoric acid; in sample \#2 low content cichoric and caffeic acid suggests a low concentration of E. purpurea; in sample \#7 the presence of caftaric acid and a comparison of the profile with that of the botanical standard E. angustifolia (Figure 1A) indicates a mixture of E. purpurea and E. angustifolia. In sample \#5 E. purpurea was identified from the distinct presence of cichoric and caftaric acid.

The results of the HPTLC chromatographic identifications of the analysed capsules yielded the following (Figure 1B and Table S4): The three samples labelled as including Echinacea sp. (\#18,\#31,\#32) contained caftaric acid and cichoric acid, and this confirmed the presence of E. purpurea. In the nine samples labelled as including specifically $E$. purpurea (samples \#19, \#22, \#23, \#26, \#27, \#28, \#29, \#30, \#33) the content of cichoric acid and caffeic acid confirmed the presence of this species, except for sample \#29. In sample \#21 labelled as including E. angustifolia the detection of echinacoside and cichoric acid and the comparison of the profile with that of the botanical standard E. angustifolia (Figure 1B) indicates the presence of a mixture of E. angustifolia and E. purpurea. In sample \#24 labelled as including E. pallida the presence of echinacoside and cichoric acid and the comparison of the profile with that of the botanical standard E. pallida indicates the presence of a mixture of E. pallida and E. purpurea (Figure 1B). In samples \#20 and \#25 labelled as including mixtures of E. purpurea and E. angustifolia, the presence of these species was confirmed by the content of echinacoside, caftaric acid, cichoric acid and caffeic acid.

The results of the HPTLC chromatographic identifications of the analysed tablets yielded the following (Figure 1C and Table S4): The two samples labelled as including Echinacea sp. (samples \#36, \#46) differ in their chromatographic profiles. Sample \#36 differs from that of the Echinacea species, and sample \#46 contains caffeic acid and cichoric acid indicating the presence of E. purpurea. For the eleven samples labelled as including E. purpurea (samples \#34, \#35, \#37, \#38, \#39, \#40, $\# 41, \# 42, \# 43, \# 44, \# 45)$ the presence of this species was confirmed in eight samples by the presence of caffeic acid and cichoric acid, and the presence of the echinacoside, caftaric acid and cichoric acid indicated a mixture of E. purpurea and E. pallida in sample \#40, as well as a low concentration of Echinacea in sample \#41. In sample \#42 no Echinacea was identified.

The results of the HPTLC chromatographic identifications of the analysed extracts yielded the following (Figure 1D and Table S4): Of the five samples labelled as including E. purpurea (samples \#47, \#49, \#51, \#52, \#53), sample \#47 was a glycerinated solution and this hampered an accurate composition estimation, sample \#53 contained very weak zones for echinacoside, caftaric acid and cichoric acid that may indicate the presence of E. angustifolia, and the presence of cichoric acid and caftaric acid in the other samples confirmed the presence of E. purpurea, however sample \#49 do not allow an accurate estimation. Sample \#48 labelled as including $E$. purpurea and E. angustifolia was also a glycerinated solution. Sample \#50 labelled as including E. pallida confirms the presence of this species.

\subsection{DNA metabarcoding}

All 53 samples had detectable DNA concentrations but the results varied from sample to sample. Fifteen samples had a concentration lower than $0.1 \mathrm{ng} / \mu 1,26$ samples ranging from 0.1 to $10 \mathrm{ng} / \mu \mathrm{l}$, and twelve higher than $10 \mathrm{ng} / \mu \mathrm{l}$. No correlation between 
obtained DNA concentration and the substrate type of the extracted product was observed (Table S5).

The raw data before demultiplexing consisted of 12,190,865 sequences, with an average of 115,008 sequences per sample for each marker. After applying our trimming and filtering quality criteria, 38 herbal products $(72 \%)$ were retained, and they were used for further analysis (Table S6). Fifteen products, including three herbal teas $(2,7,12)$ three capsules $(19,21,29)$, five tablets $(35,36,40,43,44)$ and four extracts $(47,50,51,53)$ did not yield reads or MOTUs after applying the quality filtering criteria and were excluded from further analyses. A total of 305,018 sequences passed the trimming and filtering quality criteria (2.5\% of reads), including 79,918 nrITS1 reads and 225,100 nrITS2 reads (Table S6). The MOTUs were formed using a $99 \%$ similarity clustering threshold, and 2,529 MOTUs that contained minimum 10 reads were retained and further identified using BLAST as 83 different species (Table S7). For nrITS1 we detected a total of 60 different species and 37 species on only nrITS2 (Table S7). For both, nrITS1 and nrITS2, the number of species detected per sample ranged from 1 to 19 , with an average of 5 species per sample.

[INSERT] Figure 2. Detection of Echinacea sp. using DNA metabarcoding within the herbal products per category of pharmaceutical form.

The targeted Echinacea species were detected in 34 out of 38 products ( $89 \%$ ) of the retained samples (Figure 2 and Table S8). Twenty-four (89\%) out of 27 analysed products labelled as including Echinacea sp. as the single ingredient, contained at least one Echinacea species. However only seven (26\%) contained exclusively Echinacea species, whereas the other 20 contained several other species, likely contaminants. Ten (91\%) out of 11 analysed products labelled as including a mixture of Echinacea sp. and other plant species, contained Echinacea sp. but all of them showed considerable discrepancies between ingredients listed on the label and the ones detected. The overall ingredient fidelity, meaning the proportion of the number of species indicated on the product label and the entire species diversity, as detected by DNA metabarcoding, was $89 \%$ for single ingredient products and $31 \%$ for the multiple ingredient products. The overall ingredient fidelity for all products was $43 \%$.

Per formulation category the DNA metabarcoding yielded the following results (cf. Figure 2 and Table S8): In thirteen out of 17 samples the presence of Echinacea sp. was confirmed, and in the remaining four samples, three did not yield MOTUs that passed the quality filtering criteria. The capsules contained Echinacea sp. in twelve out of 16 samples, and identification was not possible in four of the products because these did not yield MOTUs that passed the quality filtering criteria. The herbal tables contained Echinacea sp. in only six out of 13 samples, and in the remaining seven samples five did not yield MOTUs that passed the quality filtering criteria. The extracts contained Echinacea sp. in only three out of seven samples, and the remaining four did not yield MOTUs that passed the quality filtering criteria.

\section{Discussion}

The quality and authenticity of herbal products have direct impacts on their safety. Quality control must include a series of procedures to ensure the identity of the used raw materials and screening of target compounds along the value chain. 
Authentication assays in herbal production must discriminate potential adulterants and/or substitutes. However, despite the existence of well-established and widely accepted analytical methods recommended in the regulatory guidelines (EDQM, 2014; EMA, 2006) for herbal product quality assessment, their resolution and efficacy can be impeded by various factors. First, the finished herbal products are often highly processed with numerous ingredients, and even if these analytical methods are accurate in detecting specific target compounds, they have limited efficiency in detecting infrageneric substitution and do not yield any information on other plant ingredients in the products (Rossi Forim et al., 2015). Morphology based taxonomic methods are equally impeded by highly processed herbal products as these often constitute of finely powdered materials (Zhao et al., 2006).

In this study, we combined HPTLC and DNA metabarcoding. HPTLC is an more automated and reproducible form of thin-layer chromatography (TLC), and with a better separation and detection of the compounds that can be successfully used in quality control of raw materials and finished herbal products (Reich and Widmer, 2008). Reich et al. (2008) summarized a procedure for HPTLC based identification of Echinacea and other species, which was applied on 53 herbal products in this study, including herbal tea, capsules, tablets and tinctures. The results showed that each of the three targeted Echinacea species have distinguishable chromatograms within most of the herbal products (Figure 1). The main limitation of the HPLTC assay is the inability to offer insights on the presence of other plant species in the herbal product. HPTLC is less suitable to for the analysis of volatile and certain sensitive samples (Morlock and Schwack, 2010). Combination of HPTLC with methods, such us mass spectrometry (MS), ultraviolet-visible (UV-VIS) and infrared spectrometry (IR) or Fourier-transformed infrared spectroscopy (FTIR) offer further possibilities for analytical refinement in the analysis of herbal products by increasing the amount of qualitative and quantitative information. In summary, HPTLC is not the most adequate method for detection of substitution and adulteration within marketed herbal products, but it is a powerful and cost-effective method to identify specific chemotaxonomic markers, and thus applicable in the quality control of the derived herbal products.

DNA barcoding and metabarcoding are not yet validated for use in a regulatory context of quality control (Agapouda et al., 2017), but there are several studies advocating its usefulness for herbal product authentication and pharmacovigilance (Cheng et al., 2014; Coghlan et al., 2012; Ivanova et al., 2016; Newmaster et al., 2013; Raclariu et al., 2017b, 2017a). The DNA metabarcoding results in this study show that the presence of Echinacea sp. was detected in 34 (89 $\%$ ) out of 38 sequenced and retained samples. However, the results showed a lack of discriminatory resolution at the species level likely due to the low level of molecular divergence (Flagel et al., 2008; Zhang et al., 2017). Furthermore, most of the retained samples revealed a high level of discrepancy with most products not containing all the species listed on the label, but rather other off-label species. For all products, only 43 $\%$ of the species indicated on the product's label were detected species using DNA metabarcoding. Here we need to mention that the general monograph number 1433 on 'Herbal drugs' of the European Pharmacopoeia allows up to $2 \%$ foreign matter (EDQM, 2014), and that DNA metabarcoding is not a quantitative method that can be used to check if the contaminants are within this allowed range. As suggested by previous studies (Ivanova et al., 2016; Raclariu et al., 2017a), the results related to the authentication of herbal products using DNA metabarcoding need to focus primarily on checking the presence of the labeled ingredients and contaminants. The presence 
of non-listed species may be explained by various factors, including but not limited to the deliberate adulteration and unintentional substitution, that may occur starting from the early stage of the supply chain (i.e., cultivation, transport, storage), to the manufacturing process and the commercialization of the final products. DNA metabarcoding is a highly sensitive method and even traces of DNA, from grains of pollen from anemophilous species, for instance, or from plant dust in the entire manufacturing process that may accidentally contaminate the product, can be detected and identified (Raclariu et al., 2017b, 2017a). Several factors may influence the accuracy of the final results, starting with the first steps in processing the raw material to the final data analysis and interpretation. Extraction procedures, barcoding markers, primers, PCR amplification bias, high throughput sequencing library preparation, sequencing platform and trimming and filtering quality thresholds, clustering, and molecular identification algorithm all influence the final results (Pawluczyk et al., 2015; Staats et al., 2016). Moreover, public sequence reference databases, such as NCBI Genbank, pose significant challenges for reliable taxonomic affiliations, due to incorrectly identified or missing reference sequences and high levels of missing data (Hinchliff and Smith, 2014). However, at least one reference sequence of almost each genus of the known land plant is represented in NCBI GenBank (Hinchliff and Smith, 2014) and thus the use of DNA metabarcoding should be possible at least for identification at higher taxonomic levels.

As molecular genetics and sequencing technology advances, the use of DNA metabarcoding is promising for large-scale authentication of herbal products and other mixtures of economical importance. Standardization is required before DNA metabarcoding can be implemented as a routine analytical approach (Agapouda et al., 2017; Staats et al., 2016). Although, DNA metabarcoding is beneficial to authenticate species and study species diversity in complex mixture, it does not provide other essential information on target compound presence and concentration or the presence of chemical contaminations such as heavy metals, allergenic dyes and synthetic pharmaceuticals.

\section{Conclusions}

The increasing use of herbal medicines needs to be accompanied by an enhanced method for comprehensive quality control that are adequate for all the stages of the supply chain, starting from the cultivation of the raw material to the marketed herbal product. The results of our study show that HPTLC is a reliable analytical tool for routine use to identify and distinguish Echinacea species in herbal products. It allows for better separation and a course quantification of chemical constituents, but it has a limited resolution in detecting the presence of other species within the product. Here, we show that this limitation can be overcome through the complementary use of DNA metabarcoding that simultaneously confirms both the presence of the target species and of all other species, in even highly processed and multi-ingredient herbal products. Advances in sequencing technology advances make the use of DNA metabarcoding promising for large-scale authentication of herbal products. Nevertheless, standardization is required before it can be implemented as a routine complementary analytical method for regulatory quality control and herbal pharmacovigilance.

\section{Acknowledgments}


The authors wish to thank the NHM head engineers Birgitte Lisbeth Graae Thorbek and Audun Schrøder-Nielsen and the DNA lab manager Jarl Andreas Anmarkrud for the technical help and assistance with the genetic analyses. Iulia Chilat and Elena Iacob are acknowledged for their technical help and assistance with the phytochemical analyses.

Funding: This work was supported by the Romanian - EEA Research Programme operated by the MECS-ANCSI PO under the EEA Financial Mechanism 2009-2014 and project contract number $2 \mathrm{SEE} / 2014$.

Conflicts of interest: The authors declare no conflict of interests.

\section{Supporting information}

Ion-Torrent amplicon read data is deposited in DRYAD [doi pending acceptance]

\section{References}

1. Agapouda, A., Booker, A., Kiss, T., Hohmann, J., Heinrich, M., Csupor, D., 2017. Quality control of Hypericum perforatum L. analytical challenges and recent progress. J. Pharm. Pharmacol. doi:10.1111/jphp.12711

2. Altschul, S.F., Gish, W., Miller, W., Myers, E.W., Lipman, D.J., 1990. Basic local alignment search tool. J. Mol. Biol. 215, 403-410. doi:10.1016/S00222836(05)80360-2

3. Arnason, J.T., Binns, S.E., Baum, B.R., 2002. Phytochemical diversity and biological activity in Echinacea phytomedicines: challenges to quality control and germplasm improvement. Phytochem. Nutr. Health 9.

4. Barnes, J., Anderson, L.A., Gibbons, S., Phillipson, J.D., 2005. Echinacea species (Echinacea angustifolia (DC.) Hell., Echinacea pallida (Nutt.) Nutt., Echinacea purpurea (L.) Moench): a review of their chemistry, pharmacology and clinical properties. J. Pharm. Pharmacol. 57, 929-954.

5. Barnes, P.M., Bloom, B., Nahin, R.L., 2008. Complementary and alternative medicine use among adults and children: United States, 2007. Natl. Health Stat. Rep. 1-23.

6. Binns, S.E., Hudson, J., Merali, S., Arnason, J.T., 2002. Antiviral activity of characterized extracts from Echinacea spp. (Heliantheae: Asteraceae) against herpes simplex virus (HSV-I). Planta Med. 68, 780-783.

7. Brown, P.N., Chan, M., Paley, L., Betz, J.M., 2011. Determination of major phenolic compounds in Echinacea spp. raw materials and finished products by high-performance liquid chromatography with ultraviolet detection: singlelaboratory validation matrix extension. J. AOAC Int. 94, 1400-1410.

8. CAMAG, 2015. CAMAG Application notes on HPTLC identification of Echinacea (E. purpurea, E. angustifolia, E. pallida) F-24A. URL https://www.scribd.com/document/181382719/F-24a-echinacea-phenolicsHPTLC-Identification-of-Echinacea-pdf. Accessed Jun 142017.

9. Cheng, X., Su, X., Chen, X., Zhao, H., Bo, C., Xu, J., Bai, H., Ning, K., 2014. Biological ingredient analysis of traditional Chinese medicine preparation based on high-throughput sequencing: the story for Liuwei Dihuang Wan. Sci. Rep. 4, 5147. doi:10.1038/srep05147

10. Coghlan, M.L., Haile, J., Houston, J., Murray, D.C., White, N.E., Moolhuijzen, P., Bellgard, M.I., Bunce, M., 2012. Deep sequencing of plant and animal DNA 
603

604

605

606

607

608

609

610

611

612

613

614

615

616

617

618

619

contained within traditional Chinese medicines reveals legality issues and health safety oncerns. PLOS Genet 8, e1002657.

11. De Boer, H., Ichim, M.C., Newmaster, S.G., 2015. DNA barcoding and pharmacovigilance of herbal medicines. Drug Saf. 38, 611-620.

12. EAS, European Advisory Services, 2007. The use of substances with nutritional or physiological effect other than vitamins and minerals in food supplements. S https:/ec.europa.eu/food/sites/food/files/safety/docs/labelling_nutritionsupplements-2007_a540169_study_other_substances_en.pdf. Accessed June 1 2017.

13. EDQM, Council of Europe, 2014. European Pharmacopoeia. 8th Edition. Strasbourg: Council of Europe. URL https://www.edqm.eu/en/europeanpharmacopoeia-8th-edition-1563.html. Accessed March 112016.

14. EMA, European Medicine Agency 2006. Guideline on specifications: test procedures and acceptance criteria for herbal substances, herbal preparations and herbal medicinal products (EMA/CPMP/QWP/2820/00 Rev. 2). http://www.ema.europa.eu/docs/en_GB/document_library/Scientific_guideline /2011/09/WC500113210.pdf. Accessed May 222017.

15. Flagel, L.E., Rapp, R.A., Grover, C.E., Widrlechner, M.P., Hawkins, J., Grafenberg, J.L., Álvarez, I., Chung, G.Y., Wendel, J.F., 2008. Phylogenetic, morphological, and chemotaxonomic incongruence in the North American endemic genus Echinacea. Am. J. Bot. 95, 756-765. doi:10.3732/ajb.0800049

16. Gilbert, N., 2011. Regulations: Herbal medicine rule book. Nature 480, S98-S99. doi:10.1038/480S98a

17. Heitmann, K., Havnen, G.C., Holst, L., Nordeng, H., 2016. Pregnancy outcomes after prenatal exposure to echinacea: the Norwegian mother and child cohort study. Eur. J. Clin. Pharmacol. 72, 623-630.

18. Hinchliff, C.E., Smith, S.A., 2014. Some limitations of public sequence data for phylogenetic inference (in plants). PLoS ONE 9. doi:10.1371/journal.pone.0098986

19. Ivanova, N.V., Kuzmina, M.L., Braukmann, T.W.A., Borisenko, A.V., Zakharov, E.V., 2016. Authentication of herbal supplements using next-generation sequencing. PLOS ONE 11, e0156426. doi:10.1371/journal.pone.0156426

20. Kindscher, K., Wittenberg, R., 2016. The Naming and Classification of Echinacea species, in: Kindscher, K. (Ed.), Echinacea. Springer International Publishing, pp. 37-45. doi:10.1007/978-3-319-18156-1_4

21. Lammers, Y., Peelen, T., Vos, R.A., Gravendeel, B., 2014. The HTS barcode checkerpipeline, a tool for automated detection of illegally traded species from high-throughput sequencing data. BMC Bioinformatics 15, 44.

22. Loman, N.J., Misra, R.V., Dallman, T.J., Constantinidou, C., Gharbia, S.E., Wain, J., Pallen, M.J., 2012. Performance comparison of benchtop high-throughput sequencing platforms. Nat. Biotechnol. 30, 434-439. doi:10.1038/nbt.2198

23. Mistrikova, I., Vaverkova, S., 2006. Echinacea-chemical composition, immunostimulatory activities and uses. Thaiszia J Bot 16, 11-26.

24. Morlock, G., Schwack, W., 2010. Hyphenations in planar chromatography. J. Chromatogr. A, 1217, 6600-6609. doi:10.1016/j.chroma.2010.04.058

25. Newmaster, S.G., Grguric, M., Shanmughanandhan, D., Ramalingam, S., Ragupathy, S., 2013. DNA barcoding detects contamination and substitution in North American herbal products. BMC Med. 11, 222.

26. Pawluczyk, M., Weiss, J., Links, M.G., Egaña Aranguren, M., Wilkinson, M.D., Egea-Cortines, M., 2015. Quantitative evaluation of bias in PCR amplification 
and next-generation sequencing derived from metabarcoding samples. Anal. Bioanal. Chem. 407, 1841-1848.

27. Raclariu, A.C., Mocan, A., Popa, M.O., Vlase, L., Ichim, M.C., Crisan, G., Brysting, A.K., de Boer, H., 2017a. Veronica officinalis product authentication using DNA metabarcoding and HPLC-MS reveals widespread adulteration with Veronica chamaedrys. Front. Pharmacol. 8.

28. Raclariu, A.C., Paltinean, R., Vlase, L., Labarre, A., Manzanilla, V., Ichim, M.C., Crisan, G., Brysting, A.K., De Boer, H., 2017b. Comparative authentication of Hypericum perforatum herbal products using DNA metabarcoding, TLC and HPLC-MS. Sci. Rep. 7, 1291. doi:10.1038/s41598-017-01389-w

29. Reich, E., Schibli, A., Debatt, A., 2008. Validation of high-performance thin-layer chromatographic methods for the identification of botanicals in a cGMP environment. J. AOAC Int. 91, 13-20.

30. Reich, E., Widmer, V., 2008. Plant analysis 2008 - planar chromatography. Planta Med. 75, 711-718. doi:10.1055/s-0028-1088389

31. Rossi Forim, M., Perlatti, B., Soares Costa, E., Facchini Magnani, R., Donizetti de Souza, G., 2015. Concerns and considerations about the quality control of natural products using chromatographic methods. Curr. Chromatogr. 2, 20-31.

32. Schwarz, E., Metzler, J., Diedrich, J.P., Freudenstein, J., Bode, C., Bode, J.C., 2002. Oral administration of freshly expressed juice of Echinacea purpurea herbs fail to stimulate the nonspecific immune response in healthy young men: results of a double-blind, placebo-controlled crossover study. J. Immunother. 25, 413-420.

33. Schwarz, E., Parlesak, A., Henneicke-von Zepelin, H.-H., Bode, J.C., Bode, C., 2005. Effect of oral administration of freshly pressed juice of Echinacea purpurea on the number of various subpopulations of B-and T-lymphocytes in healthy volunteers: results of a double-blind, placebo-controlled cross-over study. Phytomedicine 12, 625-631.

34. Smith, T., Lynch, M., Johnson, J., Kawa, K., Bauman, H., Blumenthal, M., 2015. Herbal dietary supplement sales in US increase $6.8 \%$ in 2014. HerbalGram 107, 52-59.

35. Sperber, S.J., Shah, L.P., Gilbert, R.D., Ritchey, T.W., Monto, A.S., 2004. Echinacea purpurea for prevention of experimental rhinovirus colds. Clin. Infect. Dis. 38, 1367-1371.

36. Staats, M., Arulandhu, A.J., Gravendeel, B., Holst-Jensen, A., Scholtens, I., Peelen, T., Prins, T.W., Kok, E., 2016. Advances in DNA metabarcoding for food and wildlife forensic species identification. Anal. Bioanal. Chem. 408, 4615-4630.

37. Taberlet, P., Coissac, E., Pompanon, F., Brochmann, C., Willerslev, E., 2012. Towards next-generation biodiversity assessment using DNA metabarcoding. Mol. Ecol. 21, 2045-2050.

38. Zhang, N., Erickson, D.L., Ramachandran, P., Ottesen, A.R., Timme, R.E., Funk, V.A., Luo, Y., Handy, S.M., 2017. An analysis of Echinacea chloroplast genomes: implications for future botanical identification. Sci. Rep. 7, 216. doi:10.1038/s41598-017-00321-6

39. Zhao, Z., Hu, Y., Liang, Z., Yuen, J.P.-S., Jiang, Z., Leung, K.S.-Y., 2006. Authentication is fundamental for standardization of Chinese medicines. Planta Med. 72, 865-874. doi:10.1055/s-2006-947209 
670

671

672

673

674

675

676

677

678

679

680

681

682

683

684

685

686

687

688

689

690

691

692

693

694

695

696

\section{Figure legends}

Figure 1. High performance thin layer chromatogram (HPTLC) based identification and detection of phenylpropanoids from the Echinacea sp. herbal products. The track assignment: (1) test solutions (1-53) (2) phenylpropanoids chemical reference solutions in the following order (increasing Rf): echinacoside, cholorogenic acid, caftaric acid, cynarin, cichoric acid, caffeic acid; (3) botanical reference solution of Echinacea purpurea (root (MNR1) and aerial parts (MH1)) (4) United States Pharmacopoeia (USP) analytical reference botanical standards (E. purpurea (Spu), E. angustifolia (SA) and E. pallida (SP)) A. HPTLC chromatograms of herbal teas. B. HPTLC chromatograms of capsules. C. HPTLC chromatograms of tablets. D. HPTLC chromatograms of extracts. The chromatograms were scanned after derivatization in UV $366 \mathrm{~nm}$. Details about the herbal products can be found in the Table A.3.

Figure 2. Detection of Echinacea sp. using DNA metabarcoding within the herbal products per category of pharmaceutical form.

\section{Appendix. Supplementary materials}

Table S1 Use of medicinal Echinacea spp.

Table S2 The main chemical constituents of Echinacea spp.

Table S3 Information about the herbal products.

Table S4 HPTLC results.

Table S5 DNA and amplicon concentrations.

Table S6 Overview of the results for $99 \%$ clustering thresholds.

Table S7 HTS reads and identified MOTUs per product.

Table S8 Fidelity rate of the herbal products. 
Click here to download high resolution image

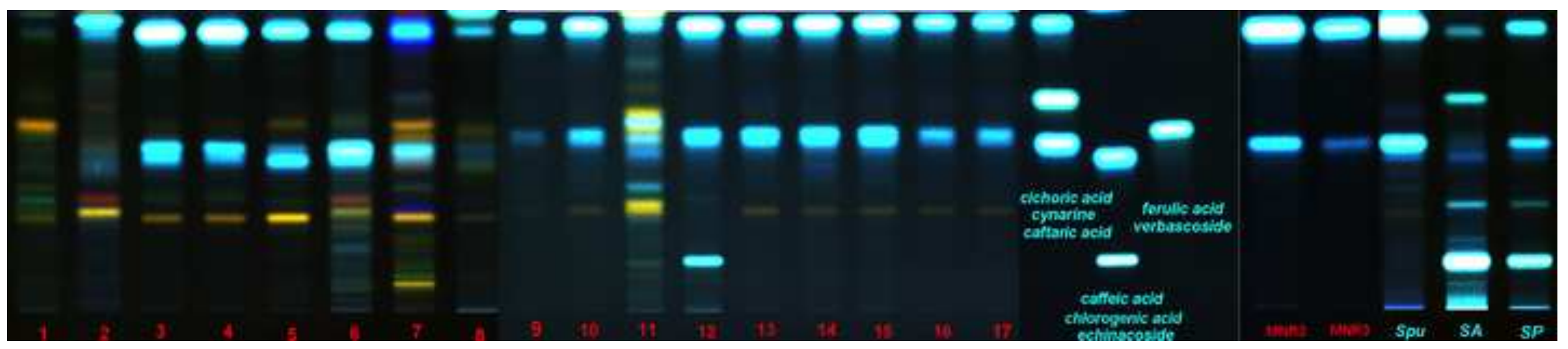


Click here to download high resolution image

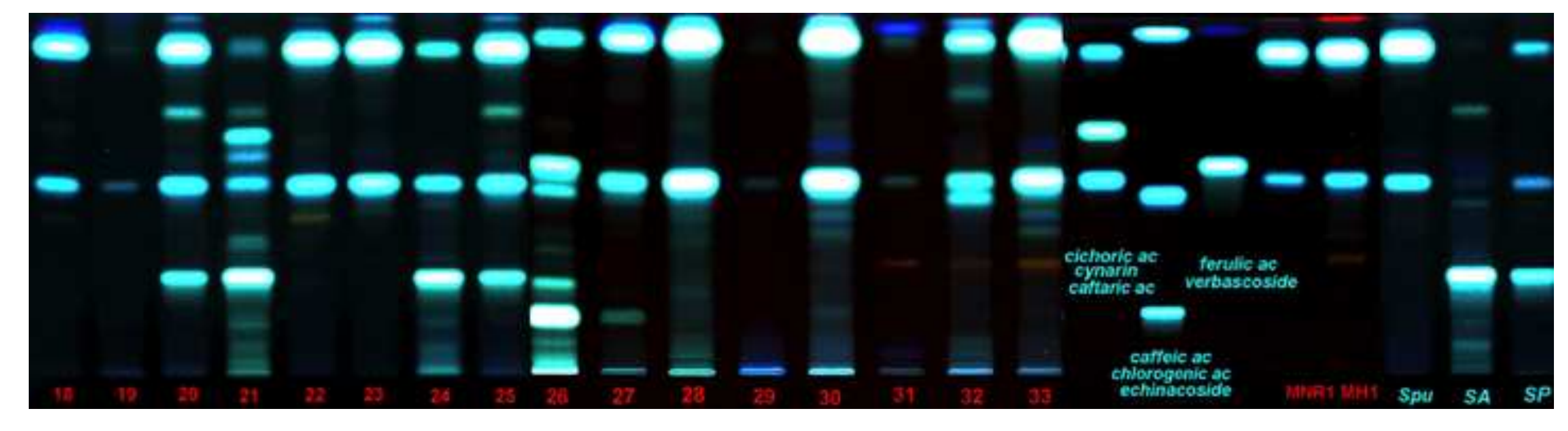

\section{Click here to download high resolution image}


Click here to download high resolution image

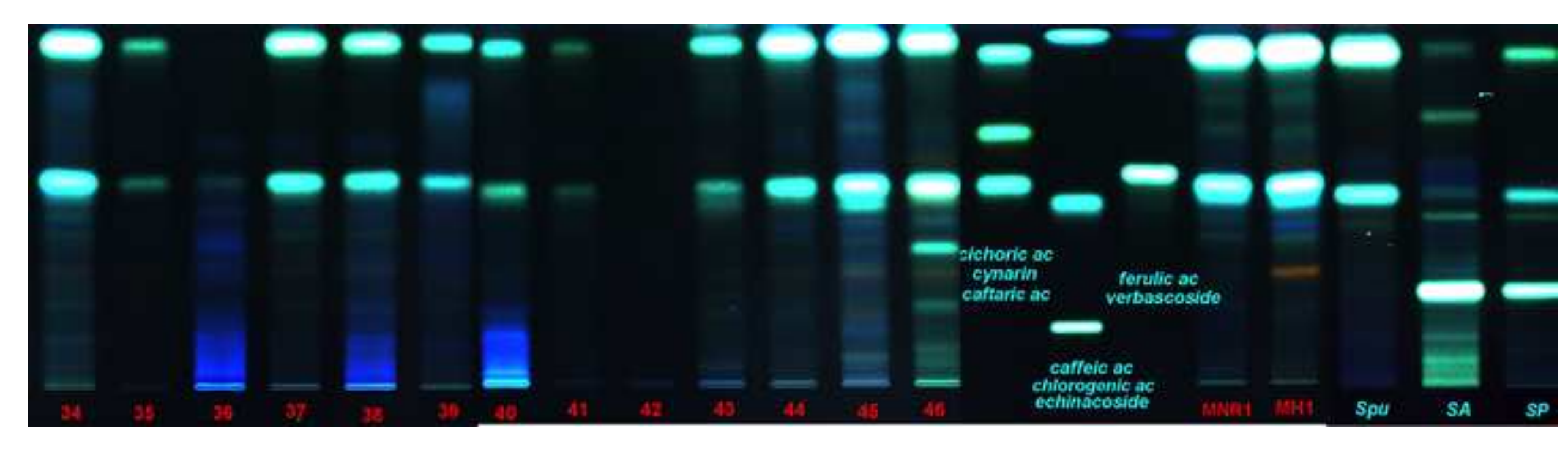

\section{click here to download high resolution image}




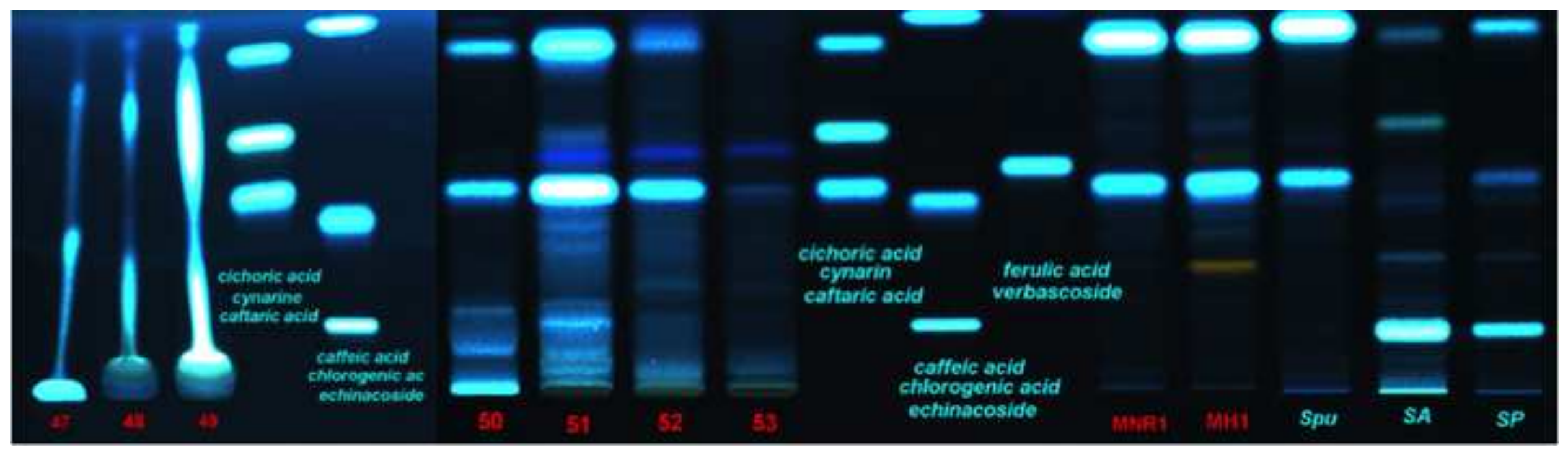


Figure 2

Products with no MOTU

$10 \% \quad 20 \% \quad 30 \%$

$50 \%$

$60 \%$

$70 \%$

$80 \%$

4

3

Herbal teas

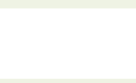

12

4

3
Tablets

Extracts
6

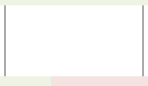

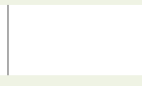
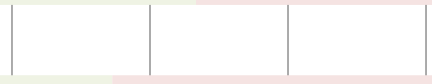

4 